qui sont altérés par un simple lavage des mains de celui qui fait la traite, lavage qui se traduit par un abaissement considérable du colititre obtenu.

Nous croyons par conséquent avoir donné un procédé très commode, simple, exact et d'application facile pour le contrôle bactériologique du lait.

(Valladolid, décembre 1935.)

\title{
LA RECHERCHE ET LE DOSAGE DE L'URINE DANS LE LAIT
}

par

\section{Dr LASCAR BURUIANA}

(Laboratoire de Chimie Biologique de la Faculté de Médecine Vétérinaire de Bucarest.)

On ne cite, jusqu'à ce joùr, aucun cas de fraude consistant le coupage du lait avec de l'urine, quoique cette falsification puisse passer inaperçue, à cause de la difficulté de la reconnaître à l'aide des méthodes employées pour déceler le coupage avec de l'eau. Pour vérifier cette affirmation nous avons préparé quelques échantillons dans lesquels le taux de l'urine était de $30 \%$ et nous avons déterminé, avant et après, les constantes suivantes : densité, indice de réfraction, et point cryoscopique. Les résultats obtenus sont donnés dans le tableau ci-dessous :

TABLEAU I.

\begin{tabular}{|c|c|c|c|c|c|c|}
\hline \multirow[b]{2}{*}{$\begin{array}{c}\text { Echantillon de lait } \\
\text { Numéros (1) }\end{array}$} & \multicolumn{2}{|c|}{ Densité } & \multicolumn{2}{|c|}{ Indice de réfraction } & \multicolumn{2}{|c|}{ Point cryoscopique } \\
\hline & Lait pur & $\begin{array}{c}\text { Lait avec } \\
30 \% \\
\text { d'urine }\end{array}$ & Lait pur & $\begin{array}{c}\text { Lait avec } \\
30 \% \\
\text { d'urine }\end{array}$ & Lait pur & $\begin{array}{c}\text { Lait avec } \\
30 \% \\
\text { d'urine }\end{array}$ \\
\hline $1 \ldots \ldots$ & 1027,2 & 1026,8 & 35,5 & 36 & $-0,51$ & $-0,90$ \\
\hline $2 \ldots \ldots \ldots \ldots$ & 1029 & 1029,3 & 38,5 & 38,7 & $-0,53$ & $-0,78$ \\
\hline $3 \ldots \ldots \ldots \ldots$ & 1031 & 1032 & 39 & 39 & $-0,51$ & $-0,72$ \\
\hline $4 \ldots \ldots \ldots$ & 1032 & 1032,5 & 38,5 & 39 & $-0,54$ & $-0,80$ \\
\hline
\end{tabular}

(1) L'échantillon (1) était falsifié avec de l’urine humaine, de densité 1023 ; les autres, avec de l'urine de vache qui, on le sait, a une densité plus forte (variations entre 1030-1045). 
L'aspect du lait mélangé avee l'urine a très peu varié. La densité et l'indice de réfraction (sérum cloro-calcique) sont restés à peu près identiques. Le point eryoscopique a varié sensiblement, mais il faut tenir compte du fait que la quantité d'urine ajoutée était assez massive.

Pour des quantités un peu au-dessous de $10 \%$, cette constante ne peut également donner aucune indication.

S'il est vrai que la falsification du lait avec de l'urine pourrait présenter seulement un intérêt théorique, le point de vue hygiénique, lui, est beaucoup plus important, La présence d'urine dans le lait, surtout en petite quantité, révèle une impureté. Une traite ou une conservation défectueuse, une manipulation ultérieure marquée par la négligence, une étable insalubre ou mal construite sont autant de causes qui favorisent les souillures du lait. C'est pour cela que le contrôle hygiénique du lait nécessite quelques fois une méthode simple et sûre pour déceler l'urine.

D'après nos connaissances, le problème concernant la recherche de l'urine dans le lait a été amorcé par Fr. MünCHENBERg dans un travail intitulé : "la valeur de la détermination de l'azote résiduel et de ses composants pour l'hygiène laitièren [1]. Dans ce travail, l'auteur, étudiant l'importance et les méthodes de la détermination de l'azote résiduel du lait, indique les applications à l'identification de l'urine. Comme conclusion, il considère que le dosage des diverses espèces d'azote : aminé, uréique, résiduel ou composants azotés (acide urique, indican), est le meilleur moyen d'identification de l'urine. En vérité, ces formes de l'azote se trouvent de loin en plus grande quantité dans l'urine que dans le lait. Dans l'ordre de sensibilité, il résulte que le dosage de l'acide urique est le plus indiqué. Celui-ci est déterminé après la défécation (tungstate de soudeacide tricloracétique) selon la méthode de BENEDIKT. On peut reconnaître des additions de $5 \%$, lorsque la valeur initiale devient deux fois plus forte (de $1 \mathrm{mgr} .55 \%$ d'acide urique à $3 \mathrm{mgr} 50 \%$ ). Suit la détermination de l'azote uréique par la méthode à l'uréase de Folin et WU. On prépare le sérum acétique, on laisse fermenter 24 heures et puis on entraîne l'ammoniaque formé pendant 3 heures. Les résultats obtenus sont médiocres, selon nous, parce qu'il faut une addition de $15 \%$ d'urine pour passer de la valeur initiale $9 \mathrm{mgr} .66 \%$ d'azote uréique à $21 \mathrm{mgr}$. $28 \%$. De plus, la méthode est longue, ce qui est un sérieux inconvénient.

La réaction de JoLLes pour l'identification de l'indican révèle quelquefois une addition de $1 \%$, mais on n'est sûr des résultats que pour des additions supérieures à $5 \%$, ainsi que nous l'avons constaté.

Ce problème nous a paru assez intéressant pour être repris. Dans le présent travail, nous rapportons une série de recherches faites 
dans le but d'augmenter, si c'est possible, la sensibilité de l'identification et de simplifier la méthode de recherche de l'urine dans le lait. Nous avons pensé que le dosage de la créatinine du lait pourrait satisfaire ces désidérata, quoique Fr. MÜNCHENBERG ait dit que cette méthode n'est pas indiquée : "weil die zur Verfügung stehenden Methoden keine praktische Erhohung des Kreatinine und Kreatininegehaltes der Milch durch Harnzusatz anzeigten. Es wurde daher von einer weiteren Untersuchung in dieser Richtung Abstand genommen."

Nous croyons que le dosage de la créatinine peut constituer une méthode particulièrement sensible parce que, dans le lait, d'après les déterminations de B. Bleyer et 0 . Kalmann [2], se trouve normalement $1 \mathrm{mgr} .2 \%$ de créatinine, tandis que l'urine en contient une quantité 110-170 fois plus grande (les déterminations faites par nous ont donné pour l'urine d'homme : 0 gr. $80-2$ gr. $80 \%$, moyenne 1 gr. $30 \%$, et pour l'urine de vache: 1 gr. $60-2$ gr. $7 \%_{0}$, moyenne 2 gr. $\left.1 \%_{0}\right)$. Dans le cas de l'acide urique et de l'urée, le rapport entre les quantités respectives, lait-urine, est seulement de 1,40. Nous sommes d'accord que la détermination de la créatinine du lait est quelque peu difficile à cause du lactose et de l'irrégularité d'action des réactifs déféquants. En vérité, la méthode de dosage colorimétrique de la créatinine repose sur la réaction à l'acide picrique et à l'hydroxide de sodium de $\mathrm{J}_{\mathrm{AFFE}}$. Dans ces conditions, le lactose réduit l'acide picrique, donnant une couleur rouge orange identique (l'artifice de W. Denis et A. S. Minot qui ajoutent, dans la solution étalon, une quantité égale de lactose n'est pas à recommander). Ce fait impose une défécation à l'aide du réactif "sulfate de cuivrehydroxide de calcium", selon VAN SLyke. Il présente toutefois l'inconvénient commun aux déféquants-sels de précipiter aussi une partie de la créatinine. B. Bleyer et 0 . KalmanN ont constaté des pertes allant jusqu'à $50 \%$. D'après nos recherches, les pertes sont plus fortes, $65 \%$ (entre 60-70\%), mais toujours les mêmes. Avec ces restrictions les dosages sont toujours comparables et très précis.

Nous décrivons plus bas une technique calquée sur celle de Folin.

\section{Dosage de la crbatinine du lait.}

a) Dans une fiole jaugée de $100 \mathrm{~cm}^{3}$ on met $20 \mathrm{~cm}^{3}$ de lait et $50 \mathrm{~cm}^{3}$ d'eau. On ajoute $10 \mathrm{~cm}^{3}$ de sulfate de cuivre à $20 \%$ et $10 \mathrm{~cm}^{3}$ d'une suspension d'hydroxide de calcium (suspension obtenue en éteignant 200 grammes de chaux vive et en complétant à 1 litre, et en agitant fortement avant usage). On ajoute de l'eau jusqu'au trait et on agite énergiquement 2-3 minutes. Cette agitation est d'une grande importance parce que l'on obtient ainsi un filtrat limpide 
en quantité suffisante et qui filtre rapidement. On laisse reposer 30 minutes et puis on filtre sur un grand papier filtre plissé. Avant la filtration on agite encore une fois.

b) On prélève du filtrat $25 \mathrm{c} \mathrm{m}^{3}$ correspondant à $5 \mathrm{~cm}^{3}$ de lait et on les met dans un ballon gradué de $50 \mathrm{~cm}^{3}$. On ajoute $5 \mathrm{~cm}^{3} \mathrm{~d}^{\prime}$ une solution saturée d'acide picrique très pur et $2 \mathrm{~cm}^{3}$ de solution d'hydroxide de sodium à $10 \%$.

On laisse 10 minutes. On complète avec de l'eau, on agite et on laisse encore 5 minutes, puis, on filtre dans une fiole très bien lavée et sèche.

On retourne sur le filtre les premières portions pour obtenir une solution parfaitement limpide. On compare au colorimètre avec une solution étalon obtenue en ajoutant, dans un ballon de $50 \mathrm{~cm}^{3}$, $1 \mathrm{c} \mathrm{m}^{3}$ d'une solution qui contient $2 \mathrm{mgr}$. \% de créatinine et en procédant comme précédemment.

Nous donnons dans le tableau II les résultats obtenus avec 10 échantillons de lait traits dans des conditions irréprochables :

TABLEAU II.

\begin{tabular}{|c|c|c|c|}
\hline & $\begin{array}{l}\text { Eehantillon } \\
\text { Numéros }\end{array}$ & $\begin{array}{l}\text { Créatinine trouvée } \\
\text { (en mgr. } \% \text { ) }\end{array}$ & $\begin{array}{c}\text { Créatinine ealculée (1) } \\
\text { (en mgr. } \%_{00} \text { ) }\end{array}$ \\
\hline 1 & $\ldots \ldots \ldots \ldots \ldots$ & 5,5 & 15,7 \\
\hline 2 & $\ldots \ldots, \ldots, \ldots$ & 6,2 & 17,7 \\
\hline 3 & $\ldots \ldots \ldots \ldots \ldots$ & 5,8 & 16,5 \\
\hline 4 & $\ldots \ldots \ldots \ldots \ldots$ & 8,0 & 22,8 \\
\hline 5 & $\ldots \ldots \ldots \ldots \ldots$ & 7,15 & 24,2 \\
\hline 6 & $\ldots \ldots \ldots \ldots \ldots$ & 6,2 & 17,1 \\
\hline 7 & $\ldots \ldots \ldots \ldots \ldots$ & 8,0 & 22,8 \\
\hline 8 & $\ldots \ldots \ldots \ldots \ldots$ & 5,9 & 16,0 \\
\hline 9 & $\ldots \ldots \ldots \ldots \ldots$ & 7,0 & 20,0 \\
\hline 10 & $\ldots \ldots \ldots \ldots \ldots \ldots$ & 8,0 & 22,8 \\
\hline \multicolumn{2}{|c|}{ Moyenne. } & 6,78 & 19,5 \\
\hline
\end{tabular}

L'appréciation colorimétrique, surtout des petites quantités de créatinine, est quelque peu délicate. C'est pour cela que nous avons recouru au photo-colorimètre élecrtique de B. LANGE. Dans ce but, nous avons déterminé le coefficient d'absorption en rapport avec une solution étalon. La courbe tracée dans le tableau III représente cette absorption en fonction de la concentration en créatinine.

Comme étalon nous avons employé la solution suivante :

On met dans un ballon gradué de $50 \mathrm{~cm}^{3}, 5 \mathrm{~cm}^{3}$ de solution

(1) Quantité absolue préexistant, avant la défécation cuivre-calcique. 
d'acide picrique et $2 \mathrm{~cm}^{3}$ de solution de $\mathrm{NaOH}$ à $10 \%$. On laisse dix minutes, on complète avec de l'eau, on agite et on laisse encore 5 minutes. On obtient une coloration ressemblant à celle donnée par la créatinine. On a une vraie solution étalon, qui correspond à une solution de créatinine de concentration infiniment petite. Avec cette solution nous avons établi le zéro du photo-colorimètre. La courbe tracée dans le tableau III donne directement la concentration en fonction de l'absorption.

TABLEAU III.

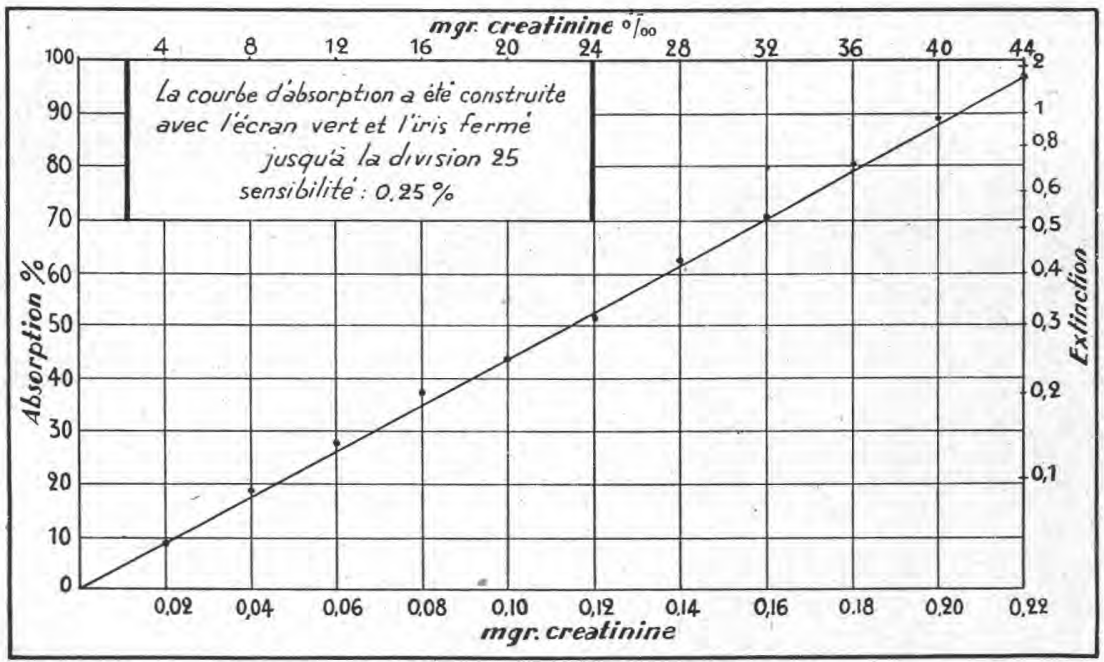

La solution de concentration inconnue a toujours une couleur plus foncée. Elle est placée, d'après la construction du colorimètre, dans la cuvette de droite. En procédant comme ci-dessus (b), on détermine la créatinine de $5 \mathrm{~cm}^{3}$ de lait. On a noté également, en abscisse, le tant pour mille.

Le procédé décrit plus haut simplifie et donne une grande sûreté aux déterminations, en ce sens, qu'il n'y a pas besoin d'une solution étalon et que n'intervient aucun facteur d'ordre subjectif comme dans la colorimétrie ordinaire à l'œil nu.

A l'aide de cette méthode nous avons essayé la méthode proposée pour la recherche de l'urine dans le lait. Nous avons préparé de nombreux échantillons avec des quantités croissantes d'urines de concentrations variables en créatinine. Les résultats obtenus en déterminant la créatinine sont enregistrés dans les tableaux suivants : 
TABLEAU IV.

\begin{tabular}{|c|c|c|c|c|c|c|c|c|c|}
\hline \multirow{3}{*}{$\begin{array}{l}\text { Contenu } \\
\text { initial du } \\
\text { lait en } \\
\text { créatinine } \\
\text { (gr. }{ }^{0 i}{ }_{00} \text { ) }\end{array}$} & \multicolumn{9}{|c|}{ Milligrammes de créatinine dans $1.000 \mathrm{~cm}^{3}$, mélange lait + urine } \\
\hline & \multicolumn{9}{|c|}{$\begin{array}{c}\text { Centimètres cubes d'urine (à } 1 \mathrm{gr} .30 \% \text { de créatinine) ajoutés à } 100 \mathrm{~cm}^{3} \\
\text { de lait }\end{array}$} \\
\hline & 1 & 2 & 3 & 4 & 5 & 6 & 7 & 8 & 9 \\
\hline 5,5 & 9,6 & 14,0 & 18,3 & 23 & 27,2 & 33 & 36 & 40,1 & 44 \\
\hline 6,2 & 9,8 & 14,8 & 19 & 24,2 & 28,4 & 34 & 37 & 24 & 45,5 \\
\hline 8,0 & 12,6 & 16,8 & 21 & 26,2 & 28,3 & 34,5 & 39 & 44 & 46,0 \\
\hline
\end{tabular}

TABLEAU V.

\begin{tabular}{|c|c|c|c|c|c|c|c|c|c|}
\hline \multirow{3}{*}{$\begin{array}{c}\text { Contenu } \\
\text { initial en } \\
\text { créatinine } \\
(\text { mgr. \% })\end{array}$} & \multicolumn{9}{|c|}{ Milligrammes de créatinine dans $1.000 \mathrm{~cm}^{3}$, mélange lait + urine } \\
\hline & \multicolumn{9}{|c|}{$\begin{array}{c}\text { Centimètres cubes d'urine (à } 1 \mathrm{gr} .6 \%{ }_{0} \text { de créatinine) ajoutés à } 100 \mathrm{~cm}^{3} \\
\text { de lait }\end{array}$} \\
\hline & 1 & 2 & 3 & 4 & 5 & 6 & 7 & 8 & 9 \\
\hline 5,8 & 11,3 & 16,5 & 21 & 27,8 & 32 & 38 & 44 & 49,5 & 55 \\
\hline 6,0 & 11,5 & 17,2 & 22,5 & 28 & 32,6 & 39 & 45 & 51 & 55,8 \\
\hline 7,1 & 13 & 19 & 24,5 & 31 & 35 & 40 & 46,1 & 52 & 57,1 \\
\hline
\end{tabular}

TABLEAU VI.

\begin{tabular}{|c|c|c|c|c|c|c|c|c|c|}
\hline \multirow{3}{*}{$\begin{array}{l}\text { Contenu } \\
\text { initial en } \\
\text { créatinine } \\
\left(\mathrm{mgr} . \%_{0}\right)\end{array}$} & \multicolumn{9}{|c|}{ Milligrammes de eréatine dans $1.000 \mathrm{~cm}^{3}$, mélange lait + urine } \\
\hline & \multicolumn{9}{|c|}{$\begin{array}{c}\text { Centimètres cubes d'urine (à } 2 \text { gr. } 1 \% \text { de créatinine) ajoutés à } 100 \mathrm{~cm}^{3} \\
\text { de lait }\end{array}$} \\
\hline & 1 & 2 & 3 & 4 & 5 & 6 & 7 & 8 & 9 \\
\hline 5,5 & 12,8 & 20,1 & 27,6 & 34,6 & 42 & 48,8 & 57 & 64 & 70 \\
\hline 6,0 & 13 & 20,7 & 28,1 & 35,5 & 42,5 & 50,2 & 58,3 & 65,2 & 71 \\
\hline 8,0 & 15,5 & 23 & 30,1 & 37,1 & 44,5 & 52,3 & 60,0 & 66,3 & 74,5 \\
\hline
\end{tabular}


TABLEAU VII.

\begin{tabular}{|c|c|c|c|c|c|c|c|c|c|}
\hline \multirow{3}{*}{$\begin{array}{l}\text { Contenu } \\
\text { initial en } \\
\text { créatinine } \\
\left(\mathrm{mgr} . \%_{0}\right)\end{array}$} & \multicolumn{9}{|c|}{ Milligrammes de créatinine dans $1.000 \mathrm{~cm}^{3}$, mélange lait + urine } \\
\hline & \multicolumn{9}{|c|}{$\begin{array}{c}\text { Centimètres cubes d'urine (à } 2 \mathrm{gr} .6 \% \text { de créatinine) ajoutés à } 100 \mathrm{~cm}^{3} \\
\text { de lait }\end{array}$} \\
\hline & 1 & 2 & 3 & 4 & 5 & 6 & 7 & 8 & 9 \\
\hline 6,0 & 15 & 24,6 & 34,1 & 42,0 & 51 & 61,0 & 70 & 79 & 90 \\
\hline 7,2 & 16,3 & . 26,0 & 34,5 & 43,1 & 52 & 63 & 72 & 79,9 & 92,1 \\
\hline 7,5 & 17 & 27 & 35,1 & 44 & 52 & 63,3 & 72,8 & 81 & 94 \\
\hline
\end{tabular}

On observe que, dans la plupart des cas, une addition de $1 \%$ d'urine suffit pour déceler avec certitude l'impureté du lait. La sensibilité de la méthode croît avec la quantité de créatinine contenue dans l'urine. Cette condition est heureusement réalisée en pratique, parce que l'urine de vache contient toujours une quantité de créatinine assez grande (en moyenne $2 \mathrm{gr} .2 \%$ ).

Or, du point de vue hygiénique, c'est cette urine qui peut constituer le plus souvent l'impureté.

La détermination de la créatinine par la méthode décrite implique un photo-colorimètre. C'est un sérieux inconvénient pour un laboratoire moins bien outillé. Le colorimètre de DUBoscQ peut donner aussi des résultats satisfaisants. Dans ce cas, on peut employer au lieu d'une solution étalon de créatinine, un échantillon de lait de propreté indiscutable, trait comme il est dit ci-dessus (b). Si l'échantillon suspect montre au colorimètre une couleur d'intensité double de celle présentée par du lait propre pris comme étalon, on peut conclure à la présence d'urine.

On voit ci-dessous les résultats obtenus.

TABLEAU VIII.

\begin{tabular}{c|c|c|c|c|c|c|c|c}
\hline \hline $\begin{array}{c}\text { Lait pur con- } \\
\text { tient : }\end{array}$ & \multicolumn{6}{|c|}{ Centimètres cubes d'urine (à 1 gr. \%o de créatinine) ajoutés aux $100 \mathrm{~cm}^{5}$} \\
de lait
\end{tabular}




\title{
CONCLUSIONS
}

1. Dans le présent travail, nous avons proposé une méthode sensible pour l'identification de l'urine dans le lait. Cette méthode repose sur le dosage de la créatinine du lait. Etant donné que l'urine contient beaucoup plus de créatinine (en moyenne 120 fois plus) que le lait, on peut déceler, avec sûreté, la présence de $1 \%$ d'urine dans le lait.

2. Nous avons perfectionné la méthode de dosage de la créatinine dans le lait en employant le colorimètre photoélectrique. Cette méthode dispense de la préparation d'une solution étalon. En construisant la courbe d'absorption en fonction de la concentration nous avons constaté que l'absorption est une fonction linéaire de la concentration, pour les petites quantités.

Le déféquant inđiqué est le mélange cuivre-calcique de VAN Slyke. Ce réactif précipite en moyenne $65 \%$ de la créatinine préexistante. Dans les mêmes conditions, ce pourcentage est particulièrement constant. Il faut tenir compte de cefacteur pour la détermination de la concentration absolue en créatinine.

3. Utilisant les données des tableaux IV-VIII, établis avec des quantités variables de lait et d'urine, on peut déterminer avec une approximation satisfaisante les additions d'urine dans le lait.

\section{BIBLIOGRAPHIE.}

[1] F. Munchenberg. Milch. Forsch. Bd. 15 H. 1 S50. 193.

[2] B. Bleyer, u. O. Kalmann. Biochem. Zeit., 153, 459, 1924.

\section{L'OBTURATION DES CRUCHES, POTS, BIDONS A LAIT}

par

\author{
Docteur G. BAR'THÉLEMI \\ Médecin-hygiéniste. M. R. San. I. \\ Laboratoire d'Hygiène de l'Université de Bruxelles.
}

Le lait récolté à l'étable est, après filtration et refroidissement éventuels quand le producteur comprend la nécessité de ces mesures, mis en cruches d'une capacité variant de 20 à 40 litres pour être transporté vers la Centrale laitière ou directement chez le détaillant.

Le transport est généralement organisé par des ramasseurs dont les camions automobiles desservent chacun une région déterminée, suivant un horaire plus ou moins bien établi.

C'est ainsi qu'on peut voir, quand on parcourt la campagne à certaines heures de la journée, les cruches posées au bord de la 